\title{
Empyema and ruptured lung abscess in adults ${ }^{1}$
}

\author{
R O W A N ICK S
}

From the Thoracic Surgical Unit, Page Chest Pavilion, Royal Prince Alfred Hospital, Melbourne

This brief annotation concerns 18 adult patients with empyema complicating the rupture of a lung abscess into the pleural cavity who were referred to my service during the period 1957 to 1962 .

By different methods of treatment all these patients have been returned to health, but in some earlier cases the illness was painful and prolonged and multiple operations were necessary to attain a final cure.

These are compared with patients treated recently in whom convalescence has been rapid, uncomplicated, and without loss of tissue.

\section{CLINICAL MATERIAL}

Particulars relevant to the treatment of 18 patients comprising the series are summarized in Table I.

\section{T A B LE I}

EMPYEMA COMPLICATING INTRAPLEURAL RUPTURE OF LUNG ABSCESS IN ADULTS, 1957 TO 1962

$$
\begin{aligned}
& \begin{array}{ccccr}
\text { Total no. of cases } & & \ldots & \ldots & 18 \\
\text { Died } \ldots & \ldots & \ldots & \ldots & 0 \\
\text { Final cure } & \ldots & \ldots & \ldots & 18
\end{array} \\
& \text { Culture: }
\end{aligned}
$$

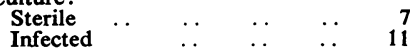

$$
\begin{aligned}
& \text { Organisms isolated } \\
& \begin{array}{llll}
\text { Pneumococcus } & & & 2 \\
\text { Staphylococcus aureus } & & \ldots & 4
\end{array} \\
& \text { Haemolytic streptococcus } \quad \ldots .2 \\
& \text { Mixed Streptococcus viridans and } \\
& \text { Staphylococcus albus } \\
& 3
\end{aligned}
$$

All patients were treated by antibiotics to which sensitivity had been ascertained. It was not possible to differentiate those in whom the abscess resulted from aspiration from those in whom it complicated a pneumonic infection.

Eleven were infected at the time of presentation and seven were sterile. From the infected cases a mixed variety of pathogenic organisms were cultured.

\section{RESULTS}

The results of treatment are summarized in Table II.

\footnotetext{
1 Delivered to the General Scientific Session, Royal Australasian
} College of Surgeons, Melbourne, in May 1963
TABLE II RESULTS OF TREATMENT

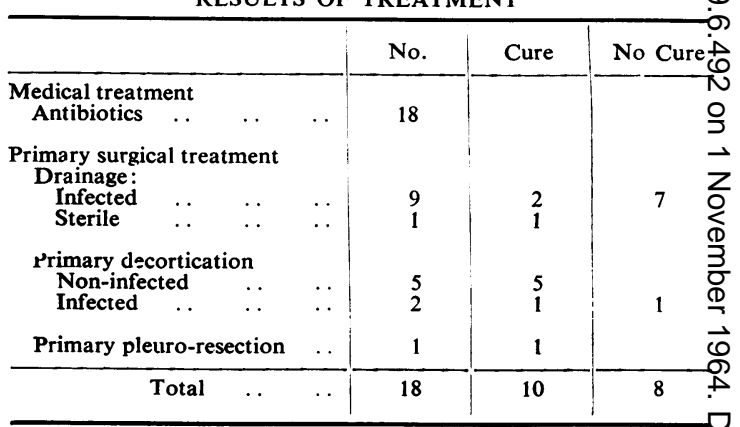

A cure followed simple drainage in three of 10 patients.

Five sterile cases treated by primary decortica tion were cured, and, of two infected cases, oneo recurred.

In the one with a localized empyema treatec $B$ by pleurolobectomy and thoracoplasty, a cure

\begin{tabular}{|c|c|c|c|c|}
\hline & No. & Cure & No & $\mathrm{Cu}$ \\
\hline 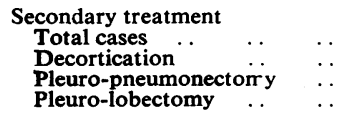 & $\begin{array}{l}8 \\
5 \\
2 \\
1\end{array}$ & $\begin{array}{l}2 \\
1\end{array}$ & & $\begin{array}{l}3 \\
1 \\
1\end{array}$ \\
\hline $\begin{array}{l}\text { Tertiary treatment } \\
\text { Saucerization, closure of fis- } \\
\text { tula and muscle graft } \\
\text { Pleuro-pneumonectomy }\end{array}$ & $\begin{array}{l}3 \\
2\end{array}$ & 3 & & 2 \\
\hline $\begin{array}{l}\text { Quarternary treatment } \\
\text { Thoracoplasty }\end{array}$ & 2 & & & 2 \\
\hline $\begin{array}{l}\text { Procedure } 5 \\
\text { Muscle graft and closure of } \\
\text { fistula } \quad . .\end{array}$ & 2 & 2 & & \\
\hline
\end{tabular}
resulted.

Primary surgery failed to achieve a cure in eight patients, and secondary procedures were performed for these (Table III).

TABLE II I

RESPONSE TO SECONDARY SURGICAL PROCEDURES

Of five patients subjected to decortication, two were cured; in both, the fistula was closed and $\vec{Q}$ the abscess cavity was marsupialized with theo 
chest wall. Of three patients submitted to pleuroresection, one of two was cured by pleuropneumonectomy and one failed with pleurolobectomy.

In tertiary treatment, saucerization of a localized empyema, followed after an interval by suture of the fistulae and the insertion of a vascular muscle graft, was finally successful in all patients.

Pleuropneumonectomy was not successful in two patients due to re-infection and to the occurrence of a broncho-pleural fistula in one of these.

In the patients referred to above, total thoracoplasty was palliative, and cure was only achieved by saucerization and closure of the fistulae by suture and muscle grafting as described (Fig. 1).

\section{COMMENT}

The rupture of a lung abscess complicating a staphylococcal pneumonia or aspiration is not an uncommon event. Convalescence is often long and complicated.

It is fortunate that the infecting organisms are sensitive to antibiotics in most cases.
It is significant that simple drainage was unsuccessful in seven of nine persistently infected cases.

Decortication was successful in five uninfected cases (including three with an open abscess cavity and fistulae at the time of operation) and in one of two infected cases. In this latter group, débridement of the necrotic abscess, closure of the fistulae, and marsupialization of the closed abscess above a soft drainage tube withdrawn through the chest wall was performed. Healing took place without complication. Treatment of one other patient treated in this way failed because of the development of osteomyelitis in a rib bared at operation.

Drainage and saucerization are wisely used as temporary procedures in doubtful cases until the infection has subsided and it is possible to study the architecture of the remaining lung.

It appears that pleurolobectomy or pleuropneumonectomy should be reserved for localized lesions with lobar or pulmonary suppuration.

Secondary closure of fistulae in healthy granulating tissue supported by a muscle graft on the surface has been found to be a safe and satisfactory operation (Fig. 1).

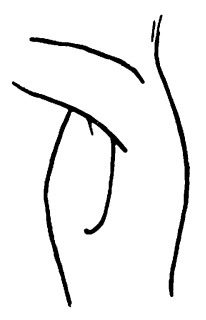

a

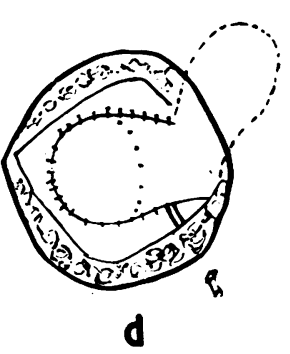

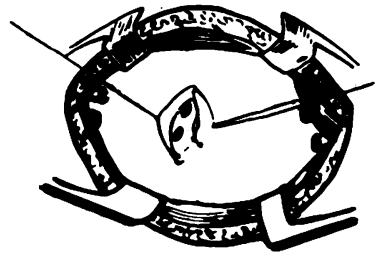

b

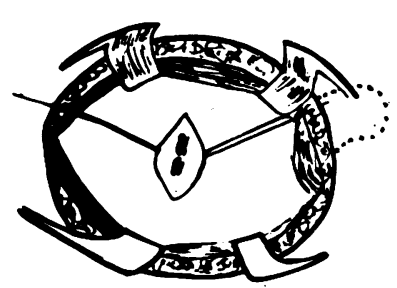

c

FIG. 1. Diagrammatic representation of operation for sealing of bronchial fistula: (a) Incision, (b) saucerization of empyema cavity, cuffing of the fistulae; (c) suture of fistulae in two layers; (d) muscle flap as a secondary seal; (e) final closure with small drainage tube in position; $(f)$ pressure pad; $(g)$ arm in sling. 


\section{PRÉCIS OF ILLUSTRATIVE CASE HISTORIES}

CASE 1 S. McH., aged 34 years. Diagnosis: Ruptured right lung abscess, débridement, decortication, and marsupialization (Fig. 2).

She was seven months pregnant when she developed right chest pain and rigors. The condition was diagnosed as pneumonia and she was treated for this with broad-spectrum antibiotics. She developed sudden dyspnoea one week later.

On examination when admitted to the Page Chest Pavilion on July 4, 1962, she was toxic and breathless. The chest radiograph showed collapse of the right lung and a pleural effusion.

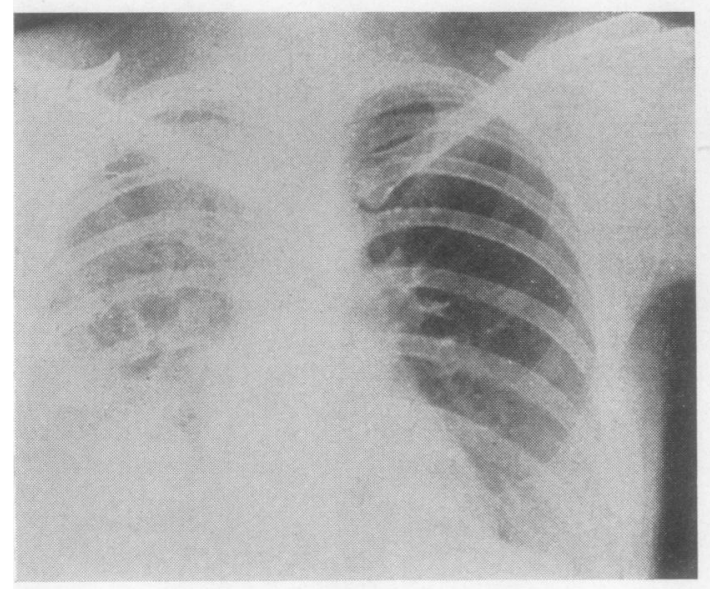

(a)

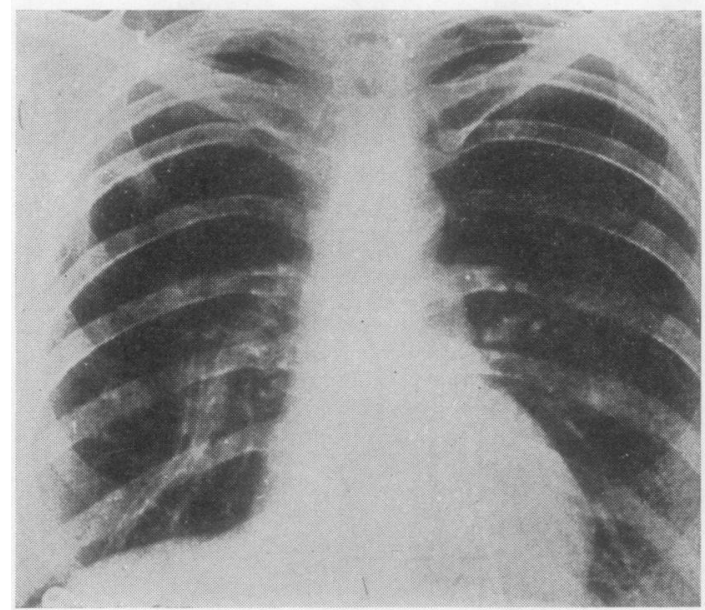

(c)
Fluid aspirated from the chest was inflammatory and sterile on culture. Ruptured hydatid cyst was suspected but the hydatid complement fixation and Casoni tests were negative. Thoracotomy performed shortly after admission revealed intrapleural rupture of a gangrenous lung abscess in the anterior segment of the right lung.

The outer necrotic abscess wall was excised and all gangrenous tissue was removed. Several large fistulae were closed with catgut, and the lung was decorticated. The abscess cavity was partially closed about a soft catheter which was withdrawn through the chest wall, and the lung was sutured to the parietal pleura at the point of exit. The chest was closed in the

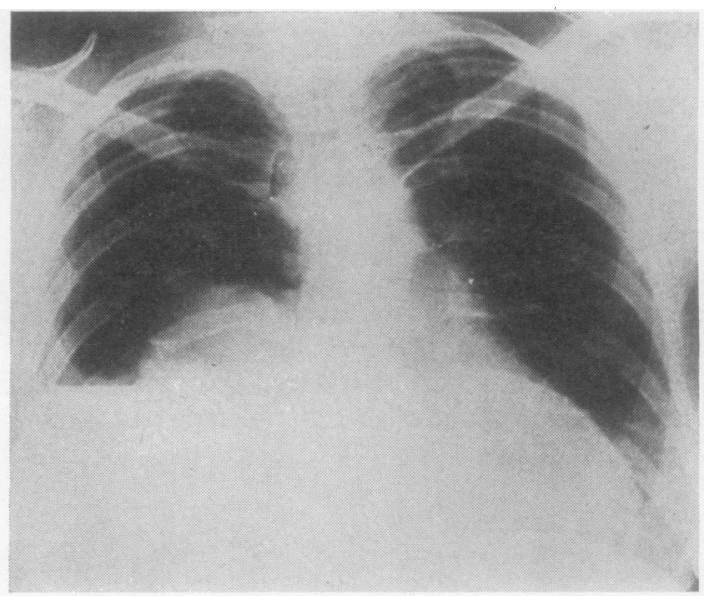

(b)

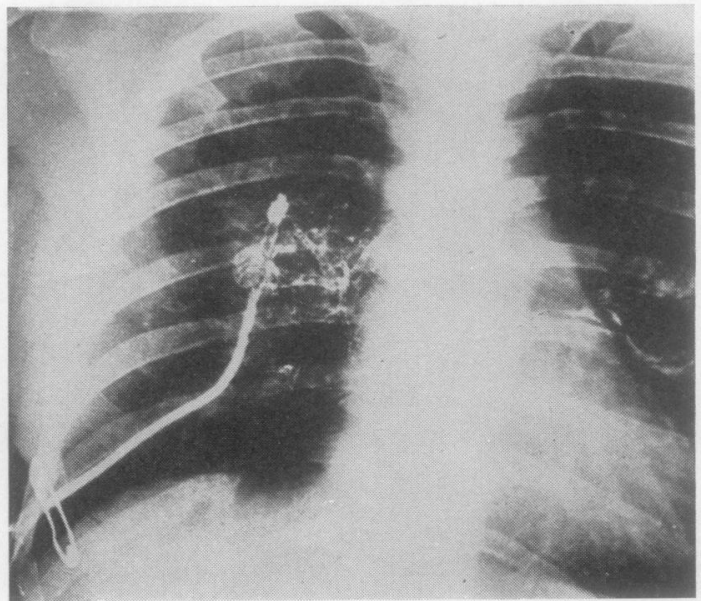

(d)

FIG. 2. Case 1. (a) Right pleuro-pneumonia with cavitation of the right upper lobe; (b) right tension pneumothorax and empyema; (c) after decortication and débridement and marsupialization. The catheter is in position; (d) sinogram showing obliteration of the cavity and small bronchial fistulae which sealed spontaneously on withdrawal. 


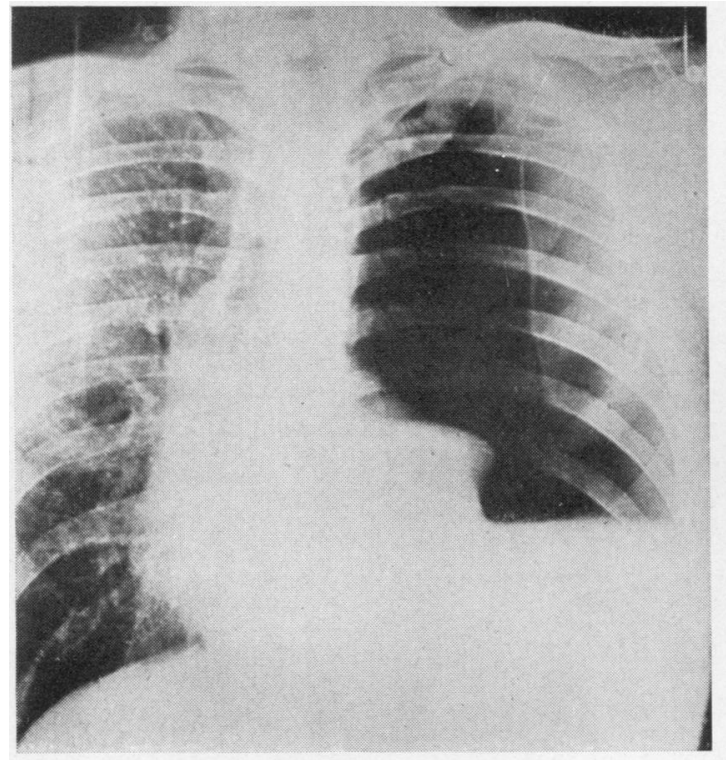

(a)

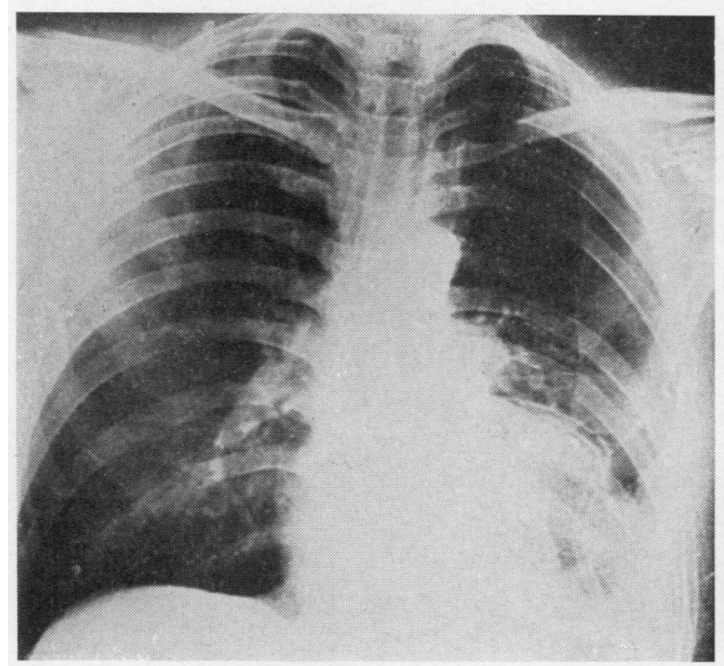

(c)

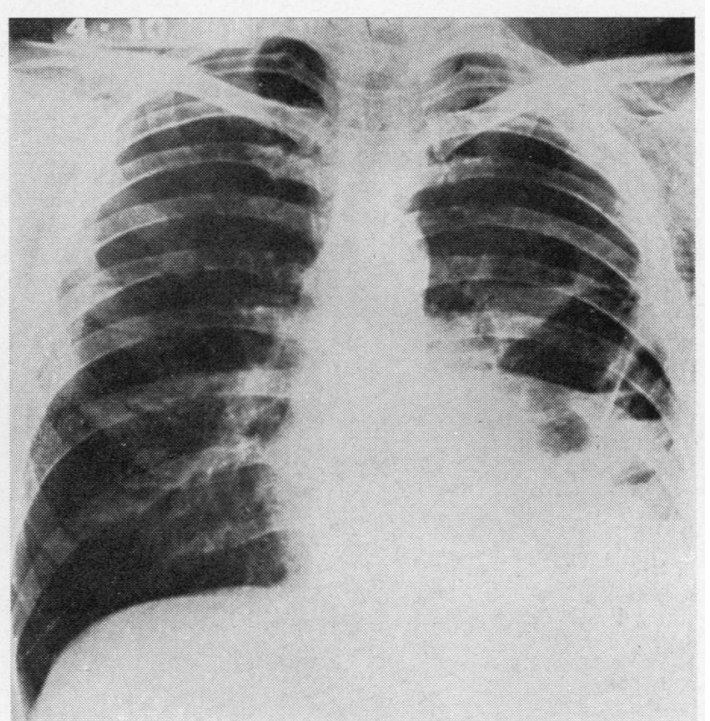

(b)

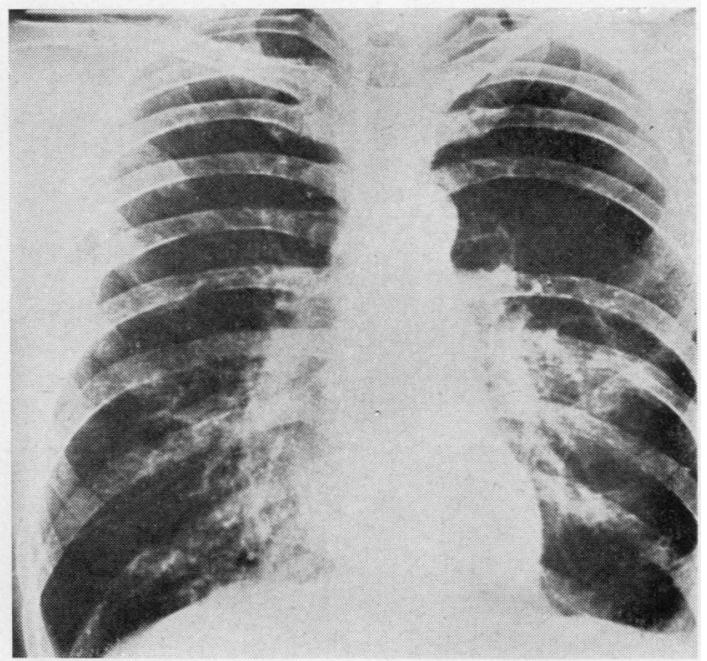

(d)

FIG. 3. Case 2. (a) Left pneumothorax and empyema; (b) recurrent pyopneumothorax; (c) after decortication, debridement, and marsupialization. The catheter is in position;

usual way with two tubes connected to underwater suction. The lung expanded and the wound healed by first intention. Premature labour pains were controlled successfully by diuadilan. The drainage from the intracavitary tube soon ceased. After verifying by a sinogram that there was no abscess cavity and that the trace merely led to small pulmonary fistulae, the tubes were removed. She remains well and without symptoms.
CASE 2 K. R., aged 40 years. Diagnosis: Ruptured lung abscess, débridement, decortication, and marsupialization (Fig. 3).

This patient was admitted to the Page Chest Pavilion on September 29, 1962, with an infected lung abscess and empyema thoracis.

He had had a pyrexial illness 10 days before admission for which he was treated by broad-spectrum antibiotics. He developed sudden dyspnoea four days 
later and was treated for spontaneous pneumothorax by an apical intercostal catheter connected to underwater drainage.

On admission the chest radiograph showed an empyema in the left lower chest with an air fluid level indicating a bronchopleural fistula. Pus grew mixed organisms from which Staphylococcus albus, Streptococcus viridans, and non-haemolytic streptococci were isolated.

At operation, apart from a thick-walled fibrous empyema, an abscess with bronchial fistulae was

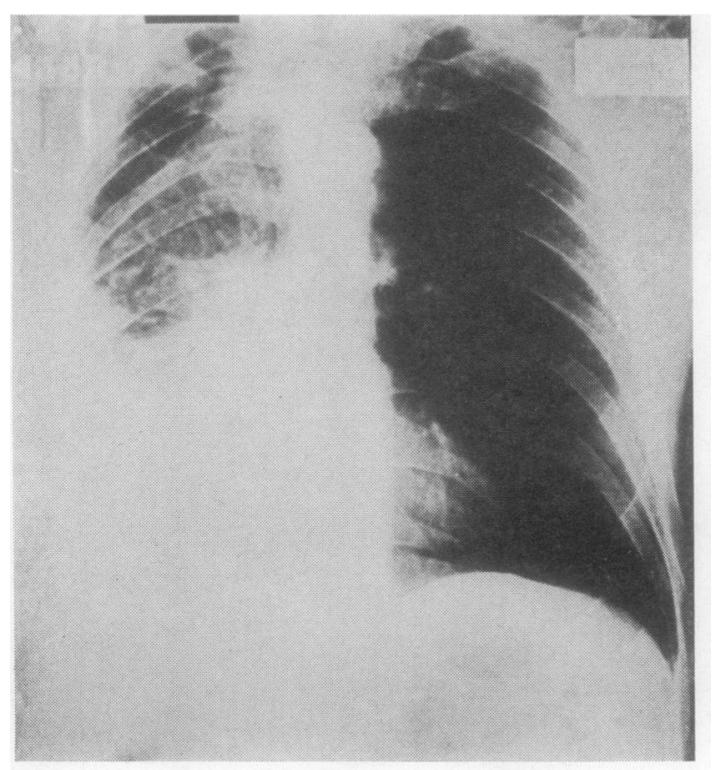

(a)

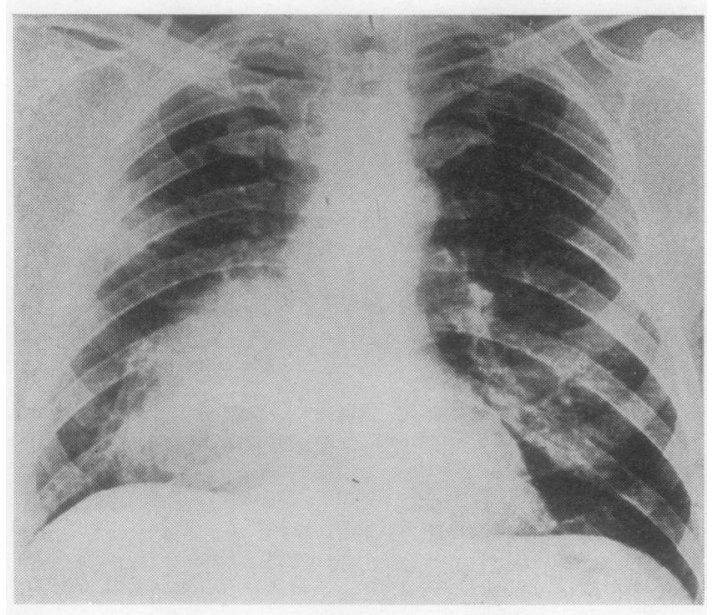

(c) found in the left lower lobe. Decortication was per- $\overline{0}$ formed, and the necrotic top of the abscess in the lower lobe was removed, the fistulae were sutured, $\overline{\bar{c}}$. and the wall was closed about a soft drainage tube $\vec{\nabla}$ which was withdrawn through the chest wall and $\propto$ sutured to the parietal pleura to prevent an escape $\%$

of air into the pleural cavity.
The chest was closed by two underwater drainage tubes. The lung expanded, and finally the marsupial- $\vec{\omega}$ ized tube was removed. Three weeks later the dis- $\sigma$ charge stopped, the culture was sterile, and the $\vec{x}$

FIG. 4. Case 3. (a) Ruptured right lung abscess with $\vec{\bullet}$ pyopneumothorax; (b) bronchogram showing abscess in oे $^{\circ}$ apical segment of the lower lobe; $(c)$ débridement and $\stackrel{t}{0}$ marsupialization; (d) final bronchogram which is essentially ${ }^{\mathrm{N}}$ normal.

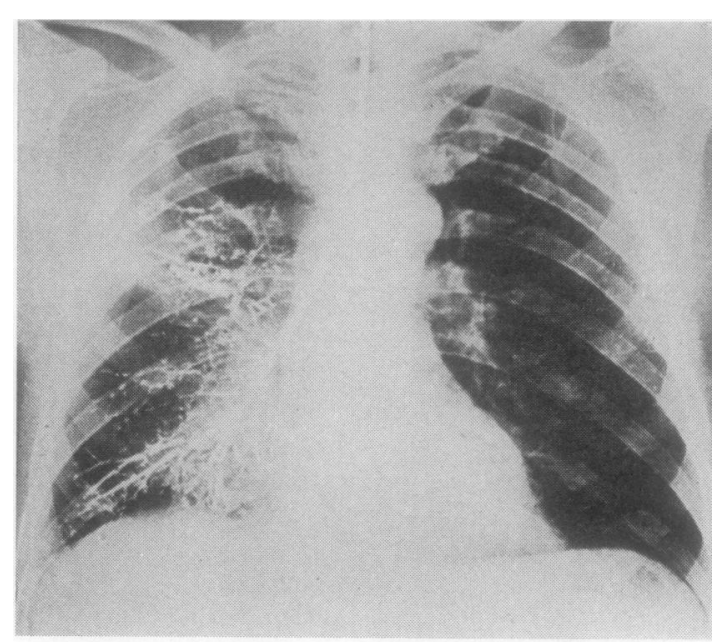

(b)

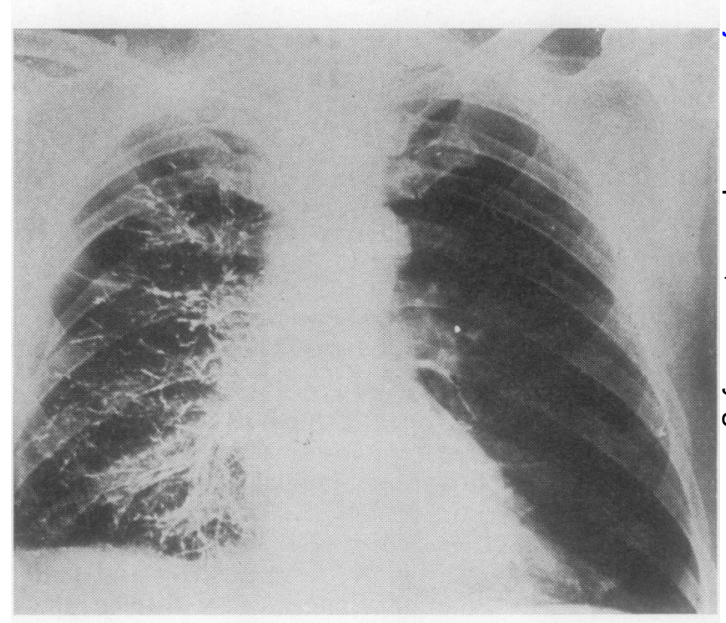

(d) 
abscess obliterated except for a small bronchocutaneous fistula. This closed when the tube was removed and has remained healed.

CASE 3 J.S., aged 55 years. Diagnosis: Ruptured lung abscess, débridement, decortication, and marsupialization (Fig. 4).

This patient was admitted to Page Chest Pavilion in November, 1957, with empyema and intrapleural rupture of an abscess of the right lower lobe of the lung, after a pneumonic attack six weeks previously. He was coughing up profuse pus. The pus aspirated from the empyema and cultures showed sparse colonies of pneumococci. He was treated with broadspectrum antibiotics and chloramphenicol.

A chest radiograph showed an empyema and consolidation of the right lower lobe. A bronchogram outlined the walls of an oval cavity in the apical segment.

At operation on December 13, 1957, there was a foul organizing fibrinous empyema cavity with thick walls and a resolving abscess of the apical segment of the lower lobe with several bronchopleural fistulae. The lung was decorticated, and, after necrotic tissue had been removed from the surface of the abscess, a soft drainage tube was stitched in place and brought through the chest wall, the point of exit from the pleura being marsupialized to the chest wall by sutures to prevent collapse of the lung. The chest was closed in the normal way with two underwater drainage tubes and suction.

Healing was uneventful and the temporary fistulae healed rapidly.

CASE 4 G. R., aged 42 years. Diagnosis: Ruptured lung abscess, débridement, decortication, and marsupialization; osteomyelitis of the ribs (Fig. 5).

This man was admitted to Page Chest Pavilion with empyema necessitatis after the intrapleural rupture of a lung abscess. He had been unwell with rigors and malaise for some weeks and had developed haemoptysis and a cough some days previously. The chest radiograph showed a total empyema, and chest aspiration revealed pus which grew anaerobic streptococci on culture.

At thoracotomy on September 13, 1958, the pleural cavity was seen to be filled with green offensive pus containing large clots of fibrin. There was an abscess $2 \mathrm{~cm}$. in diameter in the apex of the lower lobe. Partial decortication was carried out, a tube was inserted into the abscess crater, and the empyema was drained.

On January 16, 1959, he was re-admitted with recurrent empyema and chronic osteomyelitis of the rib which had been bared at operation. The overlying ribs were excised, and the overlying thick parietal pleura (up to $1 \mathrm{~cm}$.) was excised to saucerize the cavity. A large vascular muscle graft was inserted to seal off the raw area. The wound healed rapidly and has remained so. The patient is without disability.
Comment The osteomyelitis of the rib was directly related to the stripping of the periosteum at the first operation and would not have occurred if the rib had been excised. The muscle graft was successful in permanently sealing the empyema and there is now no abnormality in the lung parenchyma.

CASE 5 M. B., aged 52 years. Diagnosis: Ruptured right lung abscess, recurrent after decortication (Fig. 6).

This man was admitted to Page Chest Pavilion on October 18, 1960, with a temperature of $102^{\circ} \mathrm{F}$. He was in distress from dyspnoea and stated that he had developed a sudden chest pain at work two weeks previously. He had been treated by bed rest and chloramphenicol during this period.

The chest radiograph showed a right pneumothorax with multiple fluid levels, and aspirated pus grew a haemolytic staphylococcus on culture.

At thoracotomy on October 27, 1960, a thick-walled empyema containing fibrinous lumps was found, and decortication was performed with difficulty. The lung expanded immediately and the wound healed. Six weeks later he again developed malaise, rigors, and cough with sputum.

The chest radiograph revealed a localized cavity with a fluid level. Pyogenic staphylococci were isolated on culture of the sputum.

At operation segments of the overlying ribs were removed, and the empyema containing bronchial fistulae was saucerized. This healed and epithelialized ; but five weeks later, on July 12, 1961, persistent fistulae which were troublesome were finally sutured and supported by a muscle graft.

Comment Decortication failed in the presence of infection. The abscess was not marsupialized.

CASE 6 I. C., aged 60 years. Diagnosis: ruptured left lung abscess, saucerization and subsequent closure of fistulae and muscle graft.

This patient was admitted to Page Chest Pavilion two weeks after discharge from the Royal Prince Alfred Hospital where he had received treatment for duodenal ulceration. He had developed a cough and green purulent sputum. The chest radiograph showed a left lower empyema with an air fluid level indicating broncho-pleural fistulae. Culture of pus aspirated from the chest revealed staphylococci and scanty coliform organisms sensitive to streptomycin and chloramphenicol.

Thoracotomy on December 19, 1958, revealed a thick-walled empyema containing lumps of fibrin and a large abscess cavity in the apex of the lower lobe containing broncho-pleural fistulae.

The empyema cavity was carefully cleaned and drained by rib resection. The cavity became progressively smaller but the fistulae persisted. The cavity was saucerized. The fistulae were enclosed, and the 


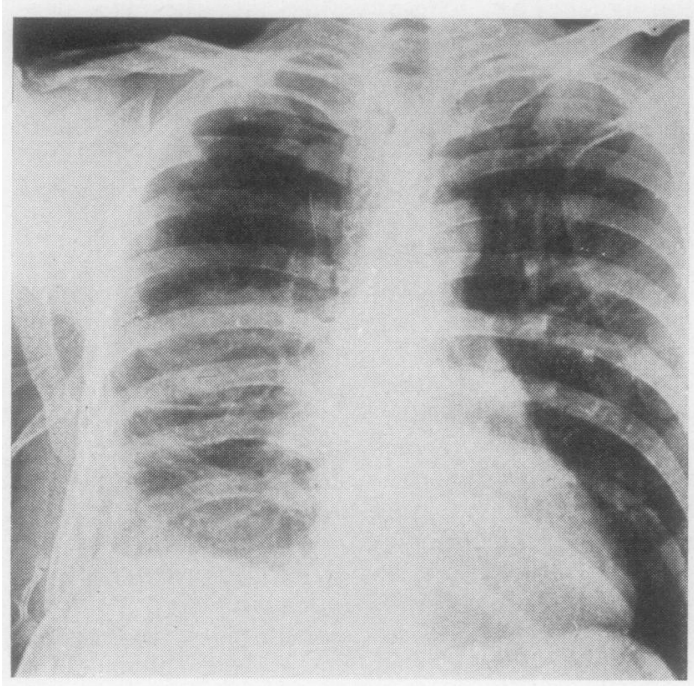

(a)

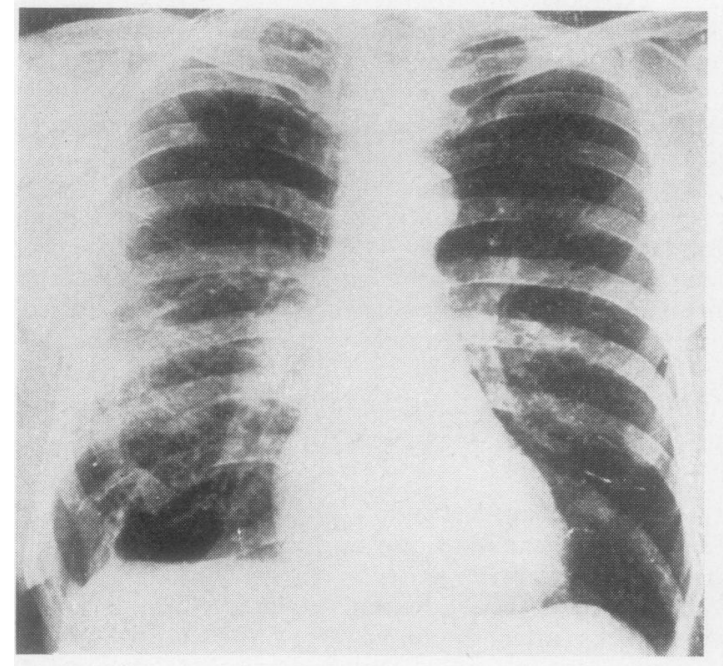

(c)

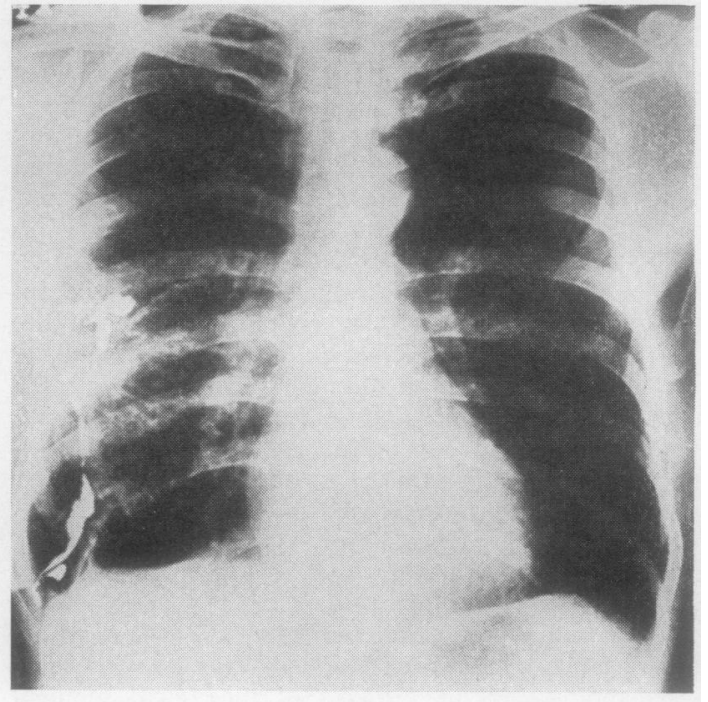

(b)

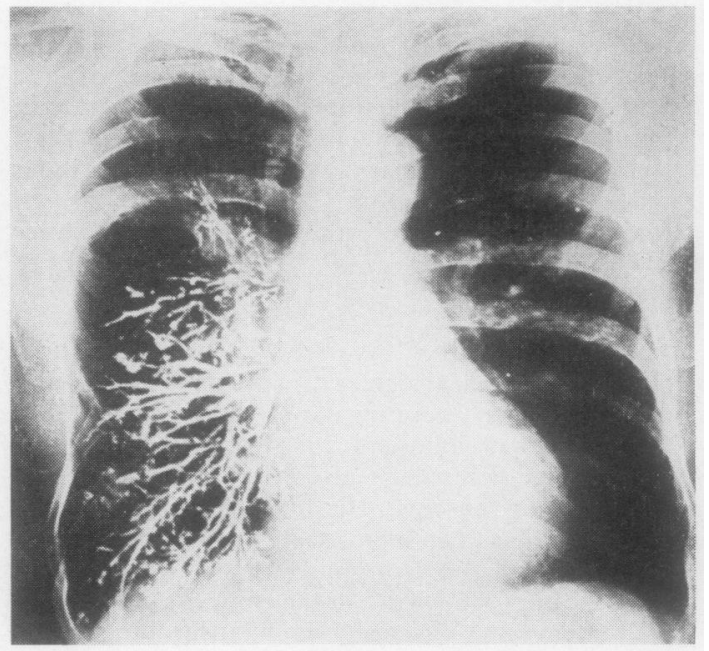

(d)

FIG. 5. Case 4. (a) Tube draining right chest, after decortication and marsupialization of abscess in right lower lobe accompanied by pyopneumothorax and empyema; (b) persistent sinus; (c) origin of sinus from the bed of the eighth rib, bared of periosteum at thoracotomy; (d) final bronchogram essentially normal.

bare granulation tissue was sealed off by a muscle graft on January 29,1959 . The wound healed rapidly and has remained healed.

Comment It is probable that this patient was suffering from an unsuspected lung abscess when he entered hospital but that this gave symptoms only when it ruptured into the pleural cavity. Relief was immediate after drainage but cure was attained only after saucerization and later closure of the fistulae supported by a muscle graft.

CASE 7 A. L., aged 46 years. Diagnosis: Ruptured lung abscess ; pleuro-lobectomy (Fig. 7).

This patient was admitted to the Page Chest Pavilion in March, 1960, with a left upper lobar abscess and localized empyema. He stated that the physical illness which had come on one month 


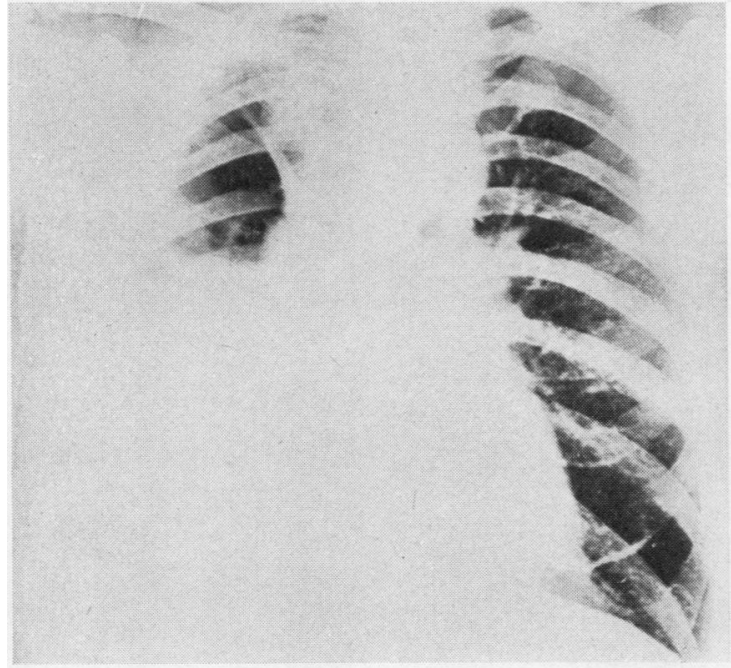

(a)

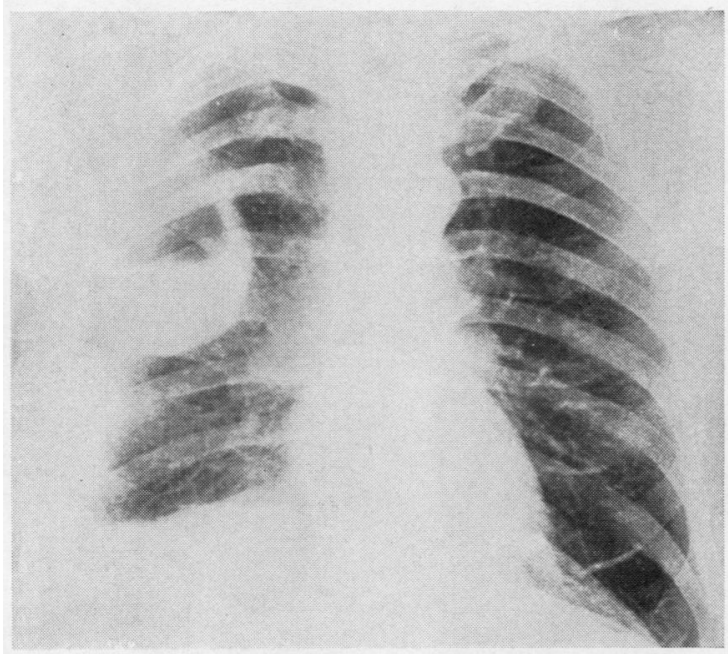

(c)

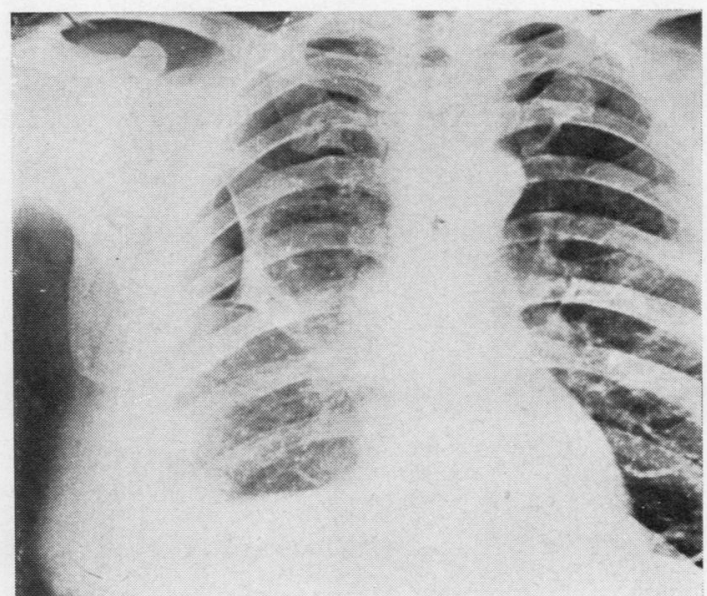

(b)

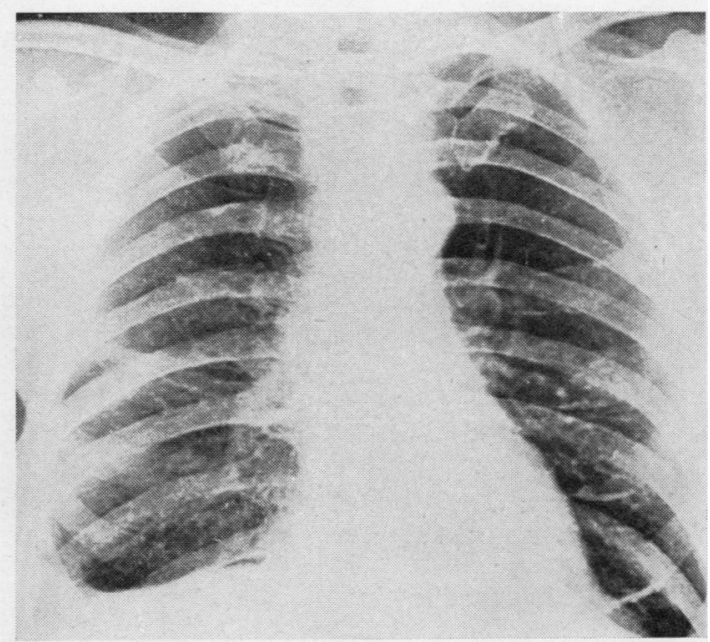

(d)

FIG. 6. Case 5. (a) Pyopneumothorax; (b) after decortication, débridement, and closure of the abscess cavity without marsupialization. Small residual pneumothorax persists; (c) after six weeks, showing recurrent empvema and bronchial fistula; (d) essentially normal chest radiograph after saucerization and delayed closure of fistulae and muscle graft.

previously had been treated by his doctor with antibiotics. During the previous two weeks he had been coughing up large amounts of pus. He was ill and drawn with a pulse rate of 100 and temperature swinging to $101^{\circ} \mathrm{F}$. The chest radiograph showed a thick-walled abscess localized to the posterior axillary segment of the right upper lobe. On culture the aspirated pus grew mixed organisms including Micrococcus catarrhalis.
At operation on April 24, 1960, a localized empyema cavity communicating with a large thickwalled abscess containing broncho-pleural fistulae was encountered. Upper pleurolobectomy was carried out with great difficulty and a thoracoplasty was performed.

He made a good recovery, but his activity remains restricted from chronic bronchitis and obstructive airway disease. 


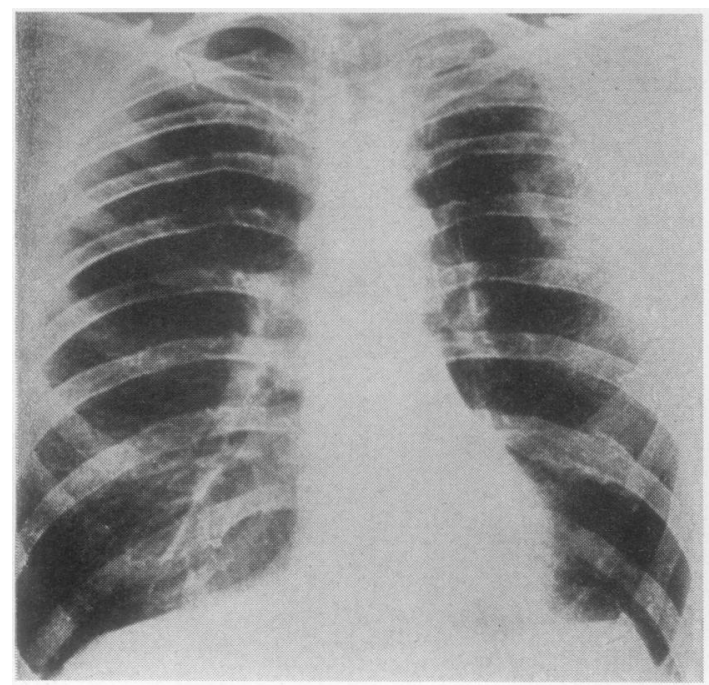

(a)

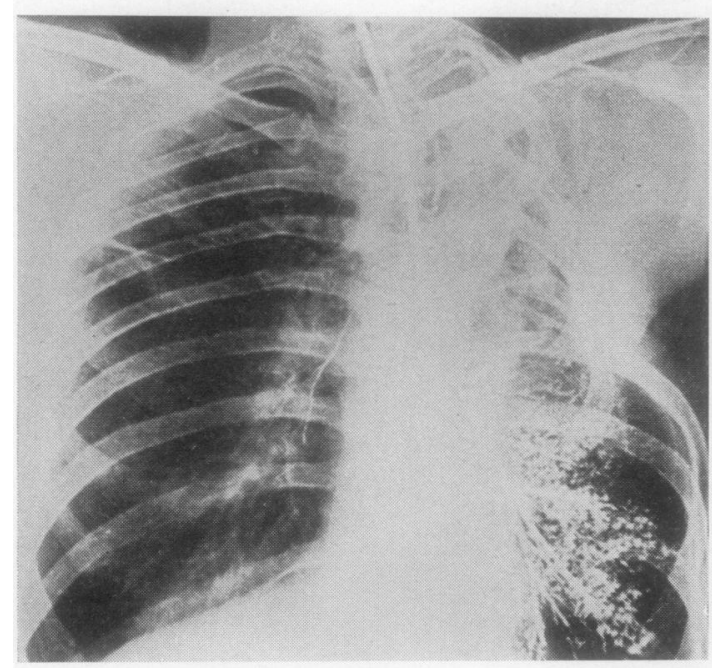

(c)

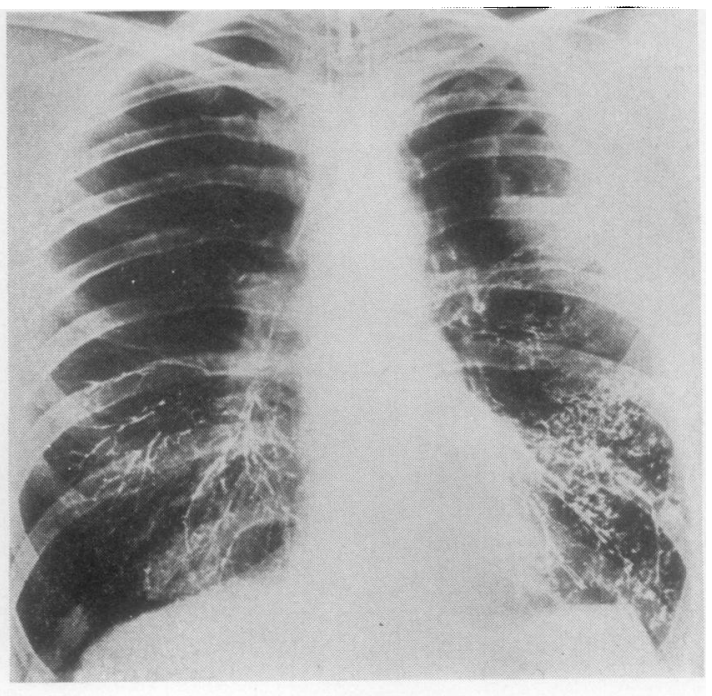

(b)

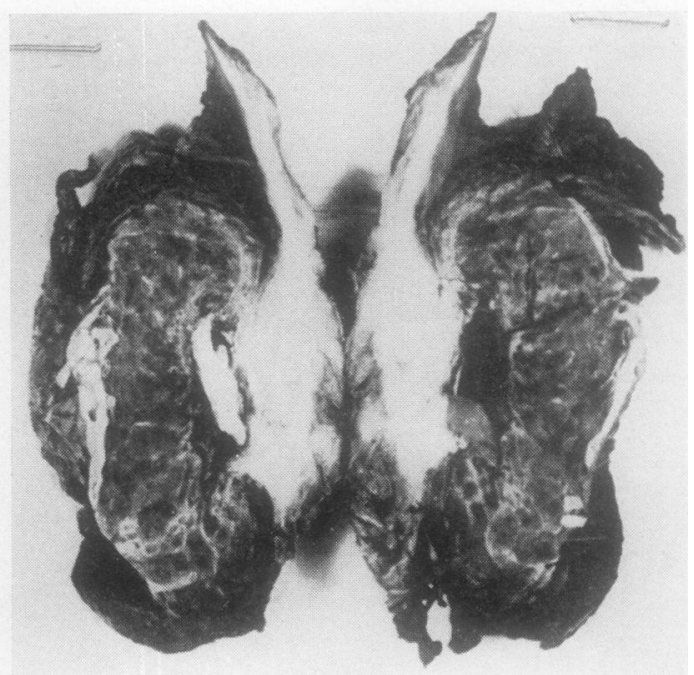

(d)

FIG. 7. Case 7. (a) Pleuro-pulmonary abscess of left upper lobe; (b) abscess with fluid level in posterior segment N of upper lobe outlined by bronchogram; $(c)$ after pleurolobectomy and thoracoplasty; (d) operation specimen of $\mathcal{N}^{\circ}$ thick-walled empyema connecting with abscess cavity.

CASE 8 S. A.. aged 66 years. Diagnosis: Pleuropneumonectomy for lung abscess and empyema complicated by tuberculosis.

This man was admitted to the Page Chest Pavilion on October 27, 1958, with an empyema and a broncho-pleural fistula after intrapleural rupture of a lung abscess. Pus was removed by pleural aspiration and grew non-haemolytic streptococci on culture. He was treated with terramycin and erythromycin. Rib resection and drainage was performed four days later. 0 Within four weeks of removal of the drainage tube the empyema recurred with pyrexia and cough with $\stackrel{\infty}{+}$ purulent sputum.

During decortication an unsuspected apical tuber- $\bar{O}$ culous cavity was opened and pleuropneumonectomy was performed.

He made a straightforward recovery and has $\varrho$ remained well since. 
Comment A permanent cure was not obtained by drainage. After opening the tuberculous cavity it was necessary to perform pleuropneumonectomy despite the risk.

A cure might have been obtained more safely by saucerization.

CASE 9 T. T., aged 47 years. Diagnosis: Ruptured gangrenous lung abscess, putrid empyema, saucerization, pleuropneumonectomy, thoracoplasty, stoma formation, and muscle graft (Fig. 8).

This man was admitted to the Page Chest Pavilion from sea; he was greatly shocked. He gave a history

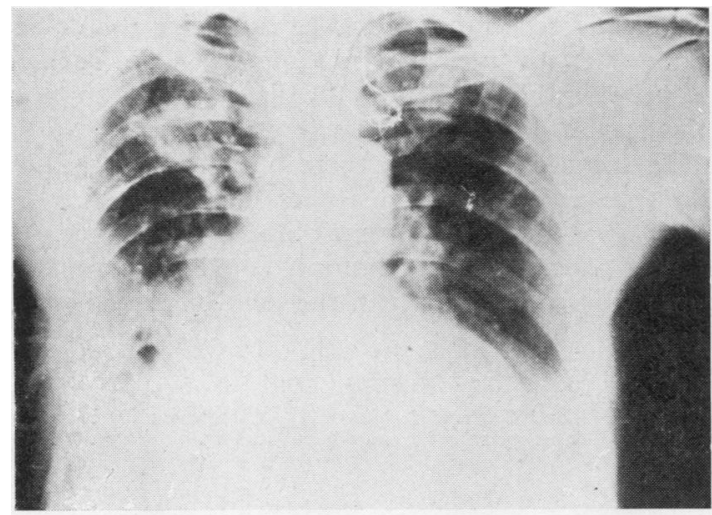

(a)

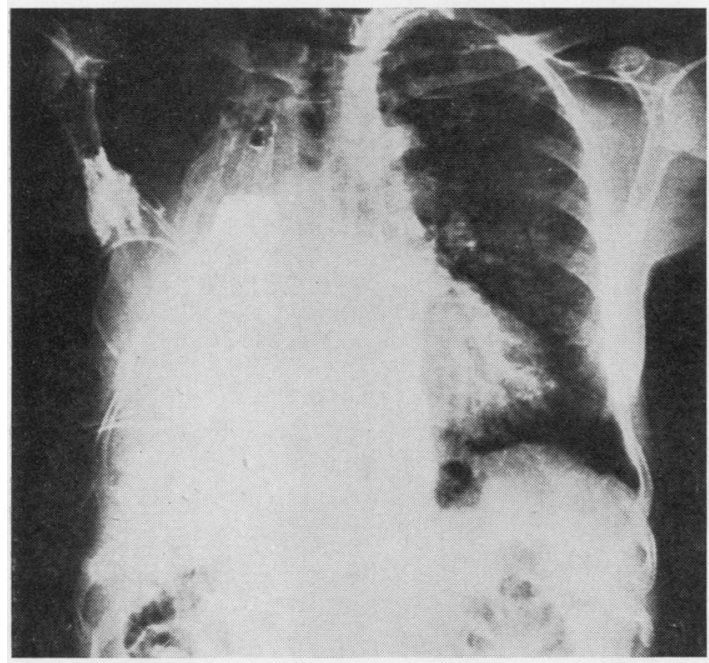

(c) of a serious illness which had developed during a voyage from India.

The chest radiograph showed an extensive opacity of the right lower zone and infiltration of the upper zone of the right lung.

Soon after admission a putrid empyema containing much gangrenous lung was evacuated and the débrided cavity was saucerized on to the skin after removal of the overlying ribs.

A Staphylococcus pyogenes infection was treated with chloramphenicol and erythromycin.

After a period of partial recovery large amounts of purulent sputum continued to be expectorated. The organism was sensitive only to erythromycin ; it was

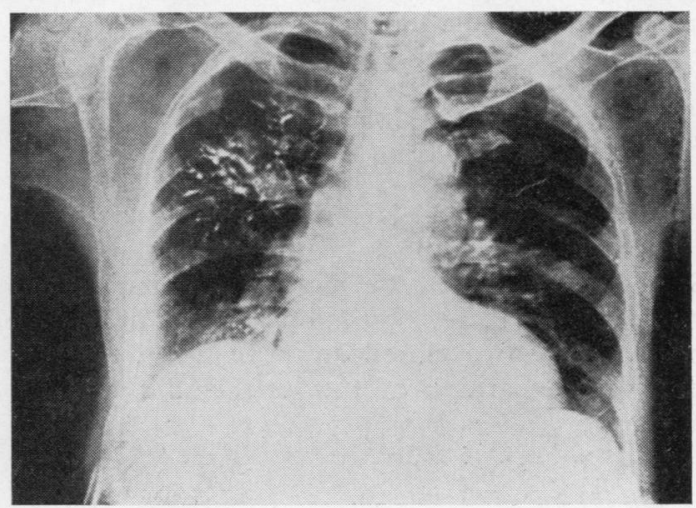

(b)

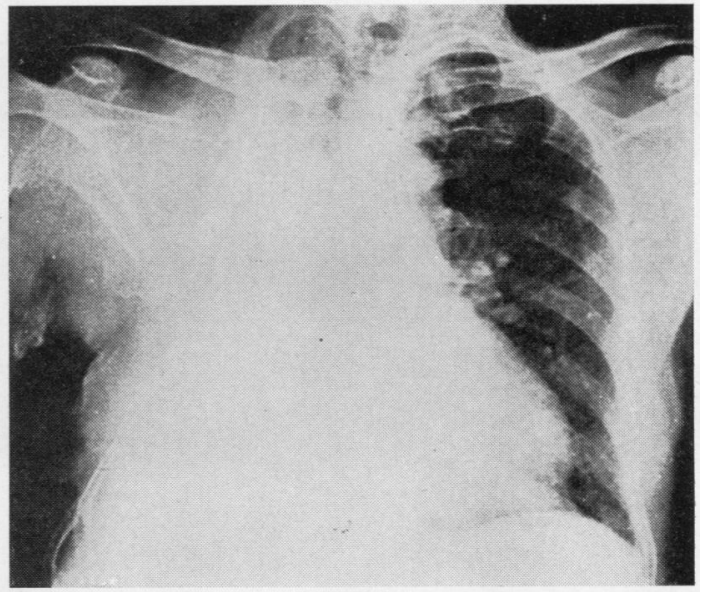

(d)

FIG. 8. Case 9. (a) Right pyopneumothorax with multiple abscesses; (b) after débridement and saucerization of a ruptured gangrenous lung abscess in the lower lobe under antibiotic cover. Residual abscess in upper lobe resistant to antibiotics; (c) total empyema after pleuropneumonectomy and thoracoplasty; (d) successful cure by large 'whale tail' muscle graft. 
resistant to penicillin, streptomycin, aureomycin, chloramphenicol, and terramycin.

It was clear that he would die unless the lung, which was shown to have residual abscesses in the upper and middle lobes as well as on the largely destroyed lower lobe, was excised. This was done successfully.

Total empyema resistant to all antibiotics supervened, and thoracoplasty was performed.

The residual cavity was widely opened by the excision of remaining osteomyelitic rib segments, and the skin was sutured to the pleura.

Three weeks later the granulating cavity was closed successfully by a giant muscle flap. The patient remained healed and free of cough and was repatriated to India three months later.

\section{SUMMARY}

In the acute stage of an infective illness with pyopneumothorax and bronchopleural fistula, it is necessary to treat the pneumonic condition and to provide local physiological conditions for recovery by two closed drainage tubes, an apical to allow expansion of the lung, and a basal for drainage.
It is not safe to perform resection in the presence of acute infection.

In the subacute stage, when the lung infection $\frac{\bar{m}}{\frac{\bar{a}}{2}}$ is controlled or is in a healing phase, it is safe $\stackrel{\mathbb{Q}}{\Omega}$ to perform decortication provided any cavity with fistulae is débrided, the fistulae closed if suitable for closure, and the residual cavity closed about a tube brought through the chest wall, the lung being sutured to the pleura at this point. If there is no residual abscess, healing will occur.

In chronic cases with localized empyema and with residual bronchial fistulae, provided the lung architecture is normal and there is no residual is lung suppuration, a satisfactory and safe surgical $N$ technique recommended is to saucerize the cavity 을 and, after an interval at a time when the surface is granulating, to suture the fistulae and support this suture line with a muscle graft.

In some cases where there has been gross lung damage and a residual abscess cavity, it may be $\underset{\Phi}{ }$ necessary to perform pleurolobectomy or pleuro-

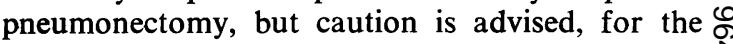

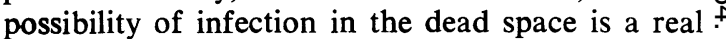
one.

\section{REFERENCES}

Pool, E. H., and Garlock, J. H. (1929) A treatment for bronchial fistula -an experin ental and clinical study. Ann. Surg., 90, 213. 